\title{
Diversity of Human Immunodeficiency Virus Type-1 Subtypes in Western Kenya
}

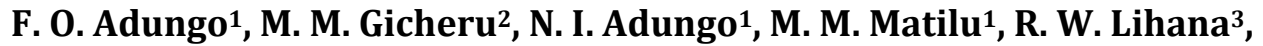 \\ S. A. Khamadi ${ }^{*}$ \\ ${ }^{1}$ Centre for Infectious and Parasitic Diseases Control Research, Kenya Medical Research Institute, Busia, Kenya \\ ${ }^{2}$ Department of Zoological Sciences, Kenyatta University, Nairobi, Kenya \\ ${ }^{3}$ Centre for Virus Research, Kenya Medical Research Institute, Nairobi, Kenya \\ Email: skhamadi@gmail.com
}

Received 10 September 2014; revised 5 October 2014; accepted 1 November 2014

Copyright (C) 2014 by authors and Scientific Research Publishing Inc.

This work is licensed under the Creative Commons Attribution International License (CC BY). http://creativecommons.org/licenses/by/4.0/

(c) (;) Open Access

\section{Abstract}

Background: HIV/AIDS is the principal pandemic in the world today. Two viral types (HIV-1 and HIV-2), with numerous groups (M, N and 0 for HIV-1 and A through $H$ for HIV-2) have emerged. These have further proliferated into numerous subtypes, sub-subtypes and circulating recombinant forms (CRF) over the last 30 years. HIV-1 variants circulate together within a geographical region providing an opportunity for recombination of viral strains within infected individuals. In Kenya, at least nine different genetic HIV-1 subtypes and several recombinant forms have been defined within group $M$, which accounts for the majority of cases in the AIDS pandemic. Objective: To determine the genetic diversity of HIV-1 in the western region of Kenya bordering Uganda. Methodology: A cross sectional study was carried out at Busia District Hospital between 2007 and 2009. A total of 75 patients were sampled randomly from a cohort of 1000 clients on antiretroviral therapy. Blood samples were analysed at the HIV Laboratory, Kenya Medical Research Institute, Nairobi, Kenya. PCR was carried out on the Pol region of HIV, sequenced and analysed by BLAST for subtypes. Results: BLAST analysis revealed the following circulating subtypes: 40/75 (53.30\%) were HIV-1 group M subtype A1; 21/75 (28.0\%) were subtype D; 5/75 (6.7\%) were subtype G; $4 / 75(5.30 \%)$ were subtype $C$; and $2 / 75(2.70 \%)$ were subtype A2. Only one isolate was identified for the other subtypes viz: $1 / 75(1.30 \%)$ resembled subtype $B ; 1 / 75(1.30 \%)$ was $\mathrm{A1} / \mathrm{C}$, and 1/75 $(1.30 \%)$ was A1/D. Conclusion: The study showed increasing HIV-1 diversity along the KenyaUganda border with the emergence of $A 1 / C$ and A1/D recombinants. Such HIV-1 diversity vis a vis the recent expanded access to antiretroviral therapy in resource limited settings calls for conti-

\footnotetext{
${ }^{*}$ Corresponding author.
} 
nuous evaluation of anti-HIV regimens. There is need therefore, for regular surveillance and monitoring for mutations that are likely to lead to drug resistance if we have to achieve successful treatment outcomes.

\section{Keywords}

HIV-1, Subtypes, Genetic Diversity

\section{Introduction}

Human Immunodeficiency Virus (HIV) infection is today one of the most devastating infections globally. Ever since the discovery of the virus some two decades ago, there has been a dramatic increase in infections and deaths due to this virus.

HIV is a member of the retroviridae family, which has a single stranded RNA genome. Two major types of HIV are currently recognised: HIV type 1 (HIV-1), and HIV type 2 (HIV-2). HIV-1 is further divided into three genetic groups: group $\mathrm{M}$ (major or main), group $\mathrm{O}$ (outlier) and group $\mathrm{N}$ (new or non-M, non-N) [1]. While groups $\mathrm{O}$ and $\mathrm{N}$ are restricted to countries of Central Africa, notably Cameroon; HIV-1 group M is widely distributed worldwide. It is responsible for the AIDS pandemic, which accounts for over $90 \%$ of HIV infections [2]. HIV-2 is restricted to countries in West Africa, where it also represents a minority of viral infections (3\% of total HIV infections). Fortunately, it is decreasing in prevalence over time [3].

\subsection{Molecular Epidemiology of HIV}

HIV-1 subtype diversity is the highest in the world. Over nine pure subtypes of HIV-1 group M are currently known (A-D, F-H, J and K). Some of these subtypes are further subdivided into sub-subtypes e.g. subtypes $\mathrm{F}$ (F1 and F2); A (A1, A2 and A3). Intrasubtype divergence of up to 20\% also occurs. Furthermore, intersubtype divergence of about $25 \%$ to $35 \%$ occurs, for the env amino acid sequences [1] [4] [5]. This intermixture of HIV-1 variants that circulate together within a geographical region provides an opportunity for recombination of virus strains within dually or multiply infected individuals [6]. Some of these recombinant forms may further achieve epidemic relevance, giving rise to unknown circulating recombinant forms (CRFs) thus complicating therapy. To date, over 40 CRFs are recognized in diverse parts of the world [7]. It is currently believed that HIV-1 M subtypes and CRFs are the result of founder effects in different geographic locales, followed by localised evolution. As a consequence, such HIV-1 forms are heterogeneously spread out worldwide [2].

\subsection{Distribution of HIV-1 Subtypes}

Africa has all HIV-1 subtypes, although A and C predominate [8]. In East Africa, subtypes A and D have dominated Uganda from mid 1980s [9]. These subtypes A and D have also been isolated from various parts of Tanzania [10]. However, in the Southern Tanzania town of Mbeya, the predominant subtype is C [11]. This is believed to have been introduced from Southern African countries where subtype C is predominant [12].

HIV-1 epidemic in Kenya like the rest of the world shows regional heterogeneity. On the basis of the env C2-V3, Neilson et al., 1999; found that subtype A predominates (71\% - 87\%), with significant components of subtype D (7\% - 29\%) and subtype C (7\% - 17\%). Carr et al., 2005 [14] has identified a full-length subtype G in Kenya. On the other hand, it has been established that HIV-1 subtypes circulating in the Northern Kenya town of Moyale mainly comprise subtype A (50\%), subtype C (39\%) and subtype D (11\%); i.e. subtypes A and C are predominant. This is probably influenced by neighbouring Ethiopia which is dominated by HIV-1 subtype C. It has been suggested that this scenario is indicative of cross-border movements influencing circulation of subtypes in northern Kenya [13]. Evidence of increasing HIV-1 diversity is emerging. For example, Lihana et al., 2006 [15] identified four circulating recombinant forms: between A1, A2, and D; A2 and D; A1 and D; A1 and G; A1 and C; A1, C and D have been documented among STI patients in Nairobi.

In the context of this increasing HIV-1 subtype diversity, it requires further studies in other parts of Kenya to monitor for mutations that are likely to lead to drug resistance if we have to achieve successful treatment out- 
comes. Thus, this study was conducted in western Kenya bordering Uganda.

\section{Materials and Methods}

\subsection{Study Site}

The study was conducted in western Kenya at sites within the border area of Kenya and Uganda. The five sites: Butula, Busia, Budalangi, Funyula and Mumias were selected to reflect representative number of patients in the cohort. This region has an adult HIV prevalence of 6.7\% (NASCOP 2007).

\subsection{Study Population and Sampling}

The study covered a cohort of 1000 patients on antiretroviral therapy supported by the Medecins Sans Frontieres (MSF) programs in Busia district hospital between 2007 and 2009. From this cohort, 75 persons were randomly selected. The samples analysed come from persons resident in the rural villages (Table 1) who, since they tend to travel less often, were very likely to have acquired their HIV infection in their villages. This facility serves patients from both Kenya and Uganda. Inclusion criteria comprised having been enrolled on antiretroviral therapy for at least 6 months.

\subsection{Blood Samples}

After obtaining informed consent, venous blood collection was performed according to the protocol previously approved by the Kenya Medical Research Institute Scientific Steering Committee and Ethical Review Board (Ref. KEMRI SSC No. 1127). At least $5 \mathrm{ml}$ of whole blood were collected into vacutainer tubes from each study subject. The blood was packed and transported to the HIV lab in Nairobi for further analysis.

\subsection{DNA Extraction}

Peripheral blood mononuclear cells (PBMCs) were extracted from whole blood by density gradient centrifugation using Ficoll-Paque Plus (Pharmacia) and DNA extracted using DNAzol (Gibco BRL ${ }^{\circledR}$ ) and ethanol precipitation. The extracted proviral DNA was used for polymerase chain reaction (PCR) amplification. A region of the HIV-1 protease gene was amplified by nested PCR. Primers NYUPOL7 (5'-GGGAATTTTCTTCAGAGCAG-3'), and NYUPOL8 (5'-TCTTCTGTCAATGGCCATTGT-3') were used in the first round and primers NYUPOL9 (5'-TCCTTAACTTCCCTCAAATCACT-3') and NYUPOL10 (5'-CTGGCACGGTTTCAATAGGACT-3') were used in the second round to amplify $297 \mathrm{bp}$ of the protease gene region corresponding to positions 2513 3209 in HIV-1 HXB2 sequence. The first round PCR was carried out in $25.0 \mu \mathrm{l}$ tube containing $2.0 \mu \mathrm{l}$ of DNA, $2.0 \mu \mathrm{l}$ of $10 \times$ buffer, $0.4 \mu \mathrm{l}$ of each forward and reverse primers (NYUPOL7 and NYUPOL8), $2.8 \mu \mathrm{l} \mathrm{MgCl}_{2}$, $10.2 \mu \mathrm{l}$ of distilled water and $0.2 \mu \mathrm{l}$ of Taq polymerase. The amplification was carried out in a thermal cycler (MJ Research, Inc). Amplification was carried out with 1 cycle of $95^{\circ} \mathrm{C}$ for $10 \mathrm{~min}$ and 35 cycles of $95^{\circ} \mathrm{C}$ for 30 $\mathrm{s}, 30^{\circ} \mathrm{C}$ for $60 \mathrm{~s}$, and $72^{\circ} \mathrm{C}$ for $1 \mathrm{~min}$, and final extension of $72^{\circ} \mathrm{C}$ for $10 \mathrm{~min}$. The nested PCR used $2.0 \mu \mathrm{l}$ of the first round PCR products as a template and used inner primers (NYUPOL9 and NYUPOL10) with the same cycling conditions. The PCR amplification was confirmed by ethidium bromide staining of samples electrophoresed on a $1.5 \%$ agarose gel.

\subsection{Sequencing and Phylogenetic Analysis}

The primers NYUPOL9 were used for PCR direct sequencing in an automated DNA sequencer (ABI 3100 Applied Biosystems, Foster City, CA) with BigDye Terminator version 3.0 Cycle Sequencing Reaction Kits (Applied Biosystems, Foster City, CA). The sequences were then aligned with previously reported HIV-1 strains of various subtypes from the Los Alamos HIV-1 database (http://hiv-web.lanl.gov) using CLUSTAL W profile alignment option. After sequencing the 75 samples, the generated nucleotides were analysed by BLAST to determine the HIV subtypes.

Genetic distances were calculated by the two-parameter method of Kumar et al., 2001 [16]. On this basis, only 64 samples were used to construct the phylogenetic tree by neighbour-joining method since the other sequences were too short (tree not shown). The reliability of the tree was estimated by 1000 bootstraps [16] [17]. The tree profile was visualized with Tree View PPC version 1.6.5. The generated sequences were deposited in 
Table 1. Distribution of genetic subtypes of hiv-1 primary isolates from the study villages.

\begin{tabular}{|c|c|c|c|c|c|}
\hline Isolate ID & $\begin{array}{l}\text { Villages of } \\
\text { residence }\end{array}$ & Sex & $\begin{array}{l}\text { Age at start of } \\
\text { HAART }\end{array}$ & $\begin{array}{c}\text { HIV-1 genetic } \\
\text { subtypes (pol region) }\end{array}$ & $\begin{array}{l}\text { Possible country of } \\
\text { origin of subtype }\end{array}$ \\
\hline Bus001 & Busia & Female & 45 & D & Uganda \\
\hline Bus002 & Busia & Female & 51 & $\mathrm{~A} 1 \mathrm{C}$ & Tanzania \\
\hline Bus003 & Busia & Female & 37 & D & Uganda \\
\hline Bus004 & Busia & Female & 45 & A1D & Kenya \\
\hline Bus005 & Busia & Female & 45 & $\mathrm{~A} 1$ & Kenya \\
\hline Bus006 & Busia & Male & 38 & $\mathrm{~A} 1$ & Uganda \\
\hline Bus007 & Busia & Male & 48 & $\mathrm{~A} 1$ & Uganda \\
\hline Bus008 & Busia & Female & 40 & A1 & Uganda \\
\hline Bus009 & Busia & Male & 43 & $\mathrm{D}$ & Uganda \\
\hline Bus010 & Busia & Female & 44 & G & DR Congo \\
\hline Bus011 & Nambale & Male & 46 & A1 & Uganda \\
\hline Bus012 & Busia & Female & 30 & A1 & Tanzania \\
\hline Bus013 & Busia & Female & 46 & $\mathrm{C}$ & Burundi \\
\hline Bus014 & Busia & Male & 32 & D & Uganda \\
\hline Bus015 & Matayos & Female & 57 & A1 & Tanzania \\
\hline Bus016 & Busia & Female & 30 & G & DR Congo \\
\hline Bus017 & Nambale & Female & 56 & A1 & Kenya \\
\hline Bus018 & Siaya/Kisumu & Female & 42 & $\mathrm{C}$ & South Africa \\
\hline Bus019 & Busia & Male & 35 & $\mathrm{~A} 1$ & Kenya \\
\hline Bus020 & Busia & Female & 52 & A1 & Kenya \\
\hline Bus021 & Busia & Male & 49 & $\mathrm{~A} 1$ & Uganda \\
\hline Bus022 & Busia & Female & 57 & A1 & Kenya \\
\hline Bus023 & Funyula & Female & 40 & A1 & Kenya \\
\hline Bus024 & Nambale & Male & 39 & A1 & Uganda \\
\hline Bus025 & Kakamega/Mumias & Female & 44 & $\mathrm{D}$ & Uganda \\
\hline Bus026 & Busia & Female & 50 & A1 & Uganda \\
\hline Bus027 & Busia & Female & 48 & $\mathrm{D}$ & Uganda \\
\hline Bus028 & Busia & Female & 45 & A1 & Kenya \\
\hline Bus029 & Busia & Male & 44 & A1 & Kenya \\
\hline Bus030 & Busia & Female & 26 & A1 & Kenya \\
\hline Bus031 & Busia & Female & 41 & $\mathrm{D}$ & Uganda \\
\hline Bus032 & Butula & Male & 36 & A1 & Kenya \\
\hline Bus033 & Busia & Male & 56 & A1 & Kenya \\
\hline Bus034 & Busia & Male & 42 & G & DR Congo \\
\hline Bus035 & Busia & Male & 36 & A1 & Uganda \\
\hline Bus036 & Busia & Female & 35 & A1 & Kenya \\
\hline Bus037 & Matayos & Female & 51 & A1 & Uganda \\
\hline
\end{tabular}




\section{Continued}

\begin{tabular}{|c|c|c|c|c|c|}
\hline Bus038 & Butula & Female & 38 & $\mathrm{D}$ & Kenya \\
\hline Bus039 & Busia & Female & 44 & $\mathrm{~A} 1$ & Kenya \\
\hline Bus040 & Busia & Male & 41 & $\mathrm{D}$ & Uganda \\
\hline Bus041 & Busia & Male & 36 & $\mathrm{D}$ & Uganda \\
\hline Bus042 & Busia & Female & 38 & $\mathrm{~A} 1$ & Kenya \\
\hline Bus043 & Bungoma & Female & 32 & $\mathrm{~A} 1$ & Kenya \\
\hline Bus044 & Busia & Female & 44 & $\mathrm{~A} 1$ & Kenya \\
\hline Bus045 & Busia & Male & 49 & $\mathrm{~A} 1$ & Uganda \\
\hline Bus046 & Nambale & Female & 36 & $\mathrm{D}$ & Uganda \\
\hline Bus047 & Bungoma & Female & 38 & G & DR Congo \\
\hline Bus048 & Busia & Female & 40 & $\mathrm{D}$ & Uganda \\
\hline Bus049 & Busia & Female & 45 & $\mathrm{D}$ & Uganda \\
\hline Bus050 & Busia & Female & 34 & $\mathrm{~A} 1$ & Kenya \\
\hline Bus051 & Bungoma & Male & 56 & $\mathrm{D}$ & Uganda \\
\hline Bus052 & Siaya/Kisumu & Male & 46 & $\mathrm{~A} 1$ & Kenya \\
\hline Bus053 & Bungoma & Female & 44 & $\mathrm{D}$ & Uganda \\
\hline Bus054 & Busia & Female & 40 & $\mathrm{~A} 1$ & Uganda \\
\hline Bus055 & Matayos & Female & 43 & $\mathrm{D}$ & Uganda \\
\hline Bus056 & Siaya/Kisumu & Male & 48 & $\mathrm{~A} 1$ & Tanzania \\
\hline Bus057 & Busia & Female & 49 & $\mathrm{D}$ & Uganda \\
\hline Bus058 & Busia & Female & 43 & $\mathrm{D}$ & Uganda \\
\hline Bus059 & Busia & Female & 52 & $\mathrm{~A} 1$ & Tanzania \\
\hline Bus060 & Bungoma & Male & 37 & $\mathrm{~A} 1$ & Kenya \\
\hline Bus061 & Busia & Female & 45 & G & DR Congo \\
\hline Bus062 & Butula & Male & 33 & $\mathrm{~A} 2$ & DR Congo \\
\hline Bus063 & Bungoma & Female & 43 & A1 & Kenya \\
\hline Bus064 & Busia & Female & 55 & $\mathrm{D}$ & Uganda \\
\hline Bus065 & Busia & Female & 32 & $\mathrm{C}$ & Burundi \\
\hline Bus066 & Siaya/Kisumu & Female & 41 & $\mathrm{D}$ & Uganda \\
\hline Bus067 & Siaya/Kisumu & Female & 45 & $\mathrm{~A} 1$ & Uganda \\
\hline Bus068 & Busia & Female & 46 & A1 & Kenya \\
\hline Bus069 & Bungoma & Female & 49 & $\mathrm{C}$ & Botswana \\
\hline Bus070 & Busia & Male & 48 & A2 & DR Congo \\
\hline Bus071 & Siaya/Kisumu & Female & 45 & $\mathrm{~A} 1$ & Uganda \\
\hline Bus072 & Matayos & Female & 33 & B & Brazil \\
\hline Bus073 & Siaya/Kisumu & Female & 33 & $\mathrm{D}$ & Uganda \\
\hline Bus074 & Budalangi & Female & 47 & $\mathrm{C}$ & Burundi \\
\hline Bus075 & Matayos & Female & 34 & $\mathrm{~A} 1$ & Uganda \\
\hline
\end{tabular}

The table shows that most of the predominant HIV-1 isolates in this border area are subytpe A1, associated with Uganda. 
the Genbank with accession numbers HQ176975-HQ177040.

\section{Results}

The most predominant HIV-1 subtype in this region was A1 (52\%) followed by subtype D (28\%) both of which are associated with Uganda. Increased HIV-1 genetic diversity is evident as shown by the six genetic subtypes (A1, D, G, C, A2, B) and two intersubtype recombinants (A1/C, and A1/D) identified (Figure 1).

The majority of the HIV-1 subtypes from this study appear to be restricted to those commonly circulating in East Africa. For instance, a total of 35 isolates from this study resembled those previously reported from Uganda in the Genbank, while 23 isolates resembled those from Kenya and only 5 isolates resembled those from Tanzania.

\section{Discussion}

In Kenya, the majority of circulating strains belong to HIV-1 group M. However, there seems to be a lot of variation, which appears to be influenced by neighbour countries. For example, Neilson et al., 1999 [18] has shown that countrywide subtype A predominates (71\% - 87\%), with significant components of subtype D (7\% 29\%) and subtype C (7\% - 17\%). Carr et al., 2005 [14] only identified subtype G. This scenario is different from that obtaining in the Northern region of Kenya town of Moyale bordering Ethiopia where on the basis of partial env sequences, Khamadi et al., 2005 [13] reported that subtype A (50\%), subtype C (39\%) and subtype D (11\%) were dominant. He attributed this to the fact that Kenya and Ethiopia share a long common border, resulting in a lot of cross-border migration, due to trade and tourist activities which may be responsible for the dynamic introduction of differing HIV subtypes and circulating recombinant forms. It must be noted that Ethiopia is dominated by HIV-1 subtype C, which interestingly is also among the dominant subtypes in Northern Kenya.

Lihana et al., 2006 [15] appears to have observed a lot of HIV-1 subtype recombinations among STI patients in Nairobi. He documented that Subtype C and D occur as non-recombinants but to a much lesser extent than subtype A. it is possible that because Nairobi is cosmopolitan, there may exist dually or multiply infected individuals which enhances opportunity for HIV-1 recombinations. These recombinations appear to be occurring recombinants between A1, A2, and D; A2 and D; A1 and D; A1 and G; A1 and C; A1, C and D.

Our study seems to support the observation that HIV-1 diversity in the border regions could be as broad as that reported in major cities. For instance, like other parts of the country the predominant circulating subtype in western Kenya is A1. However, border country influence seems to be in play in that in the Kenya-Uganda border subtype $\mathrm{D}$ is predominant, which is also the most dominant subtype in Uganda. Similarly in Moyale (KenyaEthiopia border) subtype $C$ is rampant probably due to influence from Ethiopia where subtype $C$ is dominant. Like Lihana et al., 2006 [15] reported, some HIV-1 subtype recombinations were also observed in this study. These were A1C and A1D.

It is well known that there is a lot of HIV-1 subtype diversity in the world, albeit with a degree of regional

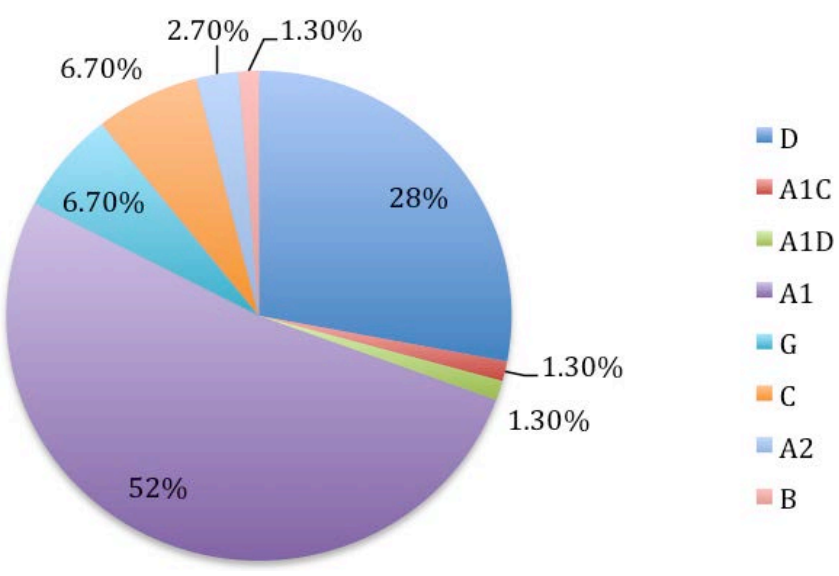

Figure 1. Pie chart of HIV-1 group m subtypes isolated from the study participants. 
segregation. Africa has all HIV-1 subtypes but A and C predominate [8]. However, in East Africa, not withstanding the continental predominance of subtypes A, there is also a significant presence of subtype D [9]. To underline the phenomenon of segregation, our study area showed subtypes A1 and D being the most common. The few pockets of subtypes $\mathrm{G}$ and $\mathrm{C}$ observed could be due to the rampant human travels and migration patterns that may lead to introduction of other HIV-1 subtypes. For example the presence of subtype G and C in our study area at the Kenya-Uganda border could probably be due to the fact that this study area is a gateway to Central Africa, notably the Congo where subtype $\mathrm{C}$ is predominant. The phenomenon of border country influence could further be supported by phylogenetic similarities of the sequenced viruses and those they were compared to from the Genbank. There was little evidence of HIV-1 subtype B in circulation which is the predominant virus circulating in Europe, and is also found in Indonesia, the Philippines, and Taiwan [19], which countries are far removed from Kenya.

\section{Conclusion}

Findings from this study reveal the complexity of HIV-1 infection in the Kenya-Uganda border region similar to those documented by Khamadi et al., 2005 [13] at the Kenya-Ethiopia border. There are continuous HIV-1 recombinations. These could portend future global pandemics, which may be characterised by even more genetically diverse viruses. There is therefore, a need for continuous review of control and treatment programs especially in the border regions. For example, surveillance studies on the extent of inter-subtype recombinations are necessary for continuous improvement of diagnosis, treatment or vaccine development. To understand further the epidemiology of HIV-1 subtype diversity in border areas, analysis of human travels, migration patterns as well as social conditions need to be done, if therapeutic interventions have to be successful.

\section{Acknowledgements}

We would like to thank the individual volunteers who made this study possible. We acknowledge the Director, Kenya Medical Research Institute for permission to publish this work.

\section{References}

[1] Robertson, D.L., Anderson, J.P., Bradac, J.A., Carr, J.K., Foley, B., Funkhouser, R.K., Gao, F., Hahn, B.H., Kalish, M.L., Kuiken, C., Learn, G.H., Leitner, T., McCutchan, F., Osmanov, S., Peeters, M., Pieniazek, D., Salminen, M., Sharp, P.M., Wolinsky, S. and Korber, B. (2000) HIV-1 Nomenclature Proposal. Science, 288, 55-56. http://dx.doi.org/10.1126/science.288.5463.55d

[2] Hemelaar, J., Gouws, E., Ghys, P.D. and Osmanov, S. (2006) Global and Regional Distribution of HIV-1 Genetic Subtypes and Recombinants in 2004. AIDS, 20, W13-W23. http://dx.doi.org/10.1097/01.aids.0000247564.73009.bc

[3] Eholie, S. and Anglaret, X. (2006) Commentary: Decline of HIV-2 Prevalence in West Africa: Good News or Bad News? International Journal of Epidemiology, 35, 1329-1330. http://dx.doi.org/10.1093/ije/dyl156

[4] Achkar, J.M., Burda, S.T., Konings, F.A., Urbanski, M.M., Williams, C.A., Seifen, D., Kahirimbanyi, M.N., Vogler, M., Parta, M., Lupatkin, H.C., Zolla-Pazner, S. and Nyambi, P.N. (2004) Infection with HIV Type 1 Group M Non-B Subtypes in Individuals Living in New York City. Journal of Acquired Immune Deficiency Syndromes, 36, 835-844. http://dx.doi.org/10.1097/00126334-200407010-00011

[5] Triques, K., Bourgeois, A., Vidal, N., Mpoudi-Ngole, E., Mulanga-Kabeya, C., Nzilambi, N., Torimiro, N., Saman, E., Delaporte, E. and Peeters, M. (2000) Near-Full-Length Genome Sequencing of Divergent African HIV Type 1 Subtype F Viruses Leads to the Identification of a New HIV Type 1 Subtype Designated K. AIDS Research and Human Retroviruses, 16, 139-151. http://dx.doi.org/10.1089/088922200309485

[6] Burke, D.S. (1997) Recombination in HIV: An Important Viral Evolutionary Strategy. Emerging Infectious Diseases, 3 , 253-259. http://dx.doi.org/10.3201/eid0303.970301

[7] Peeters, M. and Sharp, P.M. (2000) Genetic Diversity of HIV-1: The Moving Target. AIDS, 14, S129-S140.

[8] Nasioulas, G., Paraskevis, D., Magiorkinis, E., Theodoridou, M. and Hatzakis, A. (1999) Molecular Analysis of the Full-Length Genome of HIV Type 1 Subtype I: Evidence of A/G/I Recombination. AIDS Research and Human Retroviruses, 15, 745-758. http://dx.doi.org/10.1089/088922299310836

[9] Hu, D.J., Baggs, J., Downing, R.G., Pieniazek, D., Dorn, J., Fridlund, C., Biryahwaho, B., Sempala, S.D., Rayfield, M.A., Dondero, T.J. and Lal, R. (2000) Predominance of HIV-1 Subtype A and D Infections in Uganda. Emerging Infectious Diseases, 6, 609-615. http://dx.doi.org/10.3201/eid0606.000609 
[10] Blackard, J.T., Renjifo, B.R., Mwakagile, D., Montano, M.A., Fawzi, W.W. and Essex, M. (1999) Transmission of Human Immunodeficiency Type 1 Viruses with Intersubtype Recombinant Long Terminal Repeat Sequences. Virology, 254, 220-225. http://dx.doi.org/10.1006/viro.1998.9504

[11] Hoelscher, M., Kim, B., Maboko, L., Mhalu, F., von Sonnenburg, F., Birx, D.L. and McCutchan, F.E. (2001) High Proportion of Unrelated HIV-1 Intersubtype Recombinants in the Mbeya Region of Southwest Tanzania. AIDS, 15, 1461-1470. http://dx.doi.org/10.1097/00002030-200108170-00002

[12] De Baar, M.P., De Ronde, A., Berkhout, B., Cornelissen, M., Van Der Horn, K.H.M., Van Der Schoot, A.M., De Wolf, F., Lukashov, V.V. and Goudsmit, J. (2000) Subtype-Specific Sequence Variation of the HIV Type 1 Long Terminal Repeat and Primer-Binding Site. AIDS Research and Human Retroviruses, 16, 499-504. http://dx.doi.org/10.1089/088922200309160

[13] Khamadi, S.A., Ochieng, W., Lihana, R.W., Kinyua, J., Muriuki, J., Mwangi, J., Lwembe, R., Kiptoo, M., Osman, S., Lagat, N., Pelle, R., Muigai, A., Carter, J.Y., Oishi, I., Ichimura, H., Mwaniki, D.L., Okoth, F.A., Mpoke, S. and Songok, E.M. (2005) HIV Type 1 Subtypes in Circulation in Northern Kenya. AIDS Research and Human Retroviruses, 21, 810-814. http://dx.doi.org/10.1089/aid.2005.21.810

[14] Carr, J.K., Nadai, Y., Eyzaguirre, L., Saad, M.D., Khakimov, M.M., Yakubov, S.K., Birx, D.L., Graham, R.R., Wolfe, N.D., Earhart, K.C. and Sanchez, J.L. (2005) Outbreak of a West African Recombinant of HIV-1 in Tashkent, Uzbekistan. Journal of Acquired Immune Deficiency Syndromes, 39, 570-575.

[15] Lihana, R.W., Khamadi, S.A., Kiptoo, M.K., Kinyua, J.G., Lagat, N., Magoma, G.N., Mwau, M.M., Makokha, E.P., Onyango, V., Osman, S., Okoth, F.A. and Songok, E.M. (2006) HIV Type 1 Subtypes among STI Patients in Nairobi: A Genotypic Study Based on Partial pol Gene Sequencing. AIDS Research and Human Retroviruses, 22, 1172-1177. http://dx.doi.org/10.1089/aid.2006.22.1172

[16] Kumar, S., Tamura, K., Jakobsen, I.B. and Nei, M. (2001) MEGA2: Molecular Evolutionary Genetics Analysis Software. Bioinformatics, 17, 1244-1245. http://dx.doi.org/10.1093/bioinformatics/17.12.1244

[17] Rhee, S.Y., Gonzales, M.J., Kantor, R., Betts, B.J., Ravela, J. and Shafer, R.W. (2003) Human Immunodeficiency Virus Reverse Transcriptase and Protease Sequence Database. Nucleic Acids Research, 31, 298-303. http://dx.doi.org/10.1093/nar/gkg100

[18] Neilson, J.R., John, G.C., Carr, J.K., Lewis, P., Kreiss, J.K., Jackson, S., Nduati, R.W., Mbori-Ngacha, D., Panteleeff, D.D., Bodrug, S., Giachetti, C., Bott, M.A., Richardson, B.A., Bwayo, J., Ndinya-Achola, J. and Overbaugh, J. (1999) Subtypes of Human Immunodeficiency Virus Type 1 and Disease Stage among Women in Nairobi, Kenya. Journal of Virology, 73, 4393-4403.

[19] Ortiz, M., Muñoz, L., Bernal, A., Rodriguez, A., Zorraquino, A., Vadillo, J., Salas, A., Moreno, A. and García-Sáiz, A. (2000) Molecular Characterization of Non-B HIV Type 1 Subtypes from Africa in Spain. AIDS Research and Human Retroviruses, 16, 1967-1971. http://dx.doi.org/10.1089/088922200750054693 
Scientific Research Publishing (SCIRP) is one of the largest Open Access journal publishers. It is currently publishing more than 200 open access, online, peer-reviewed journals covering a wide range of academic disciplines. SCIRP serves the worldwide academic communities and contributes to the progress and application of science with its publication.

Other selected journals from SCIRP are listed as below. Submit your manuscript to us via either submit@scirp.org or Online Submission Portal.
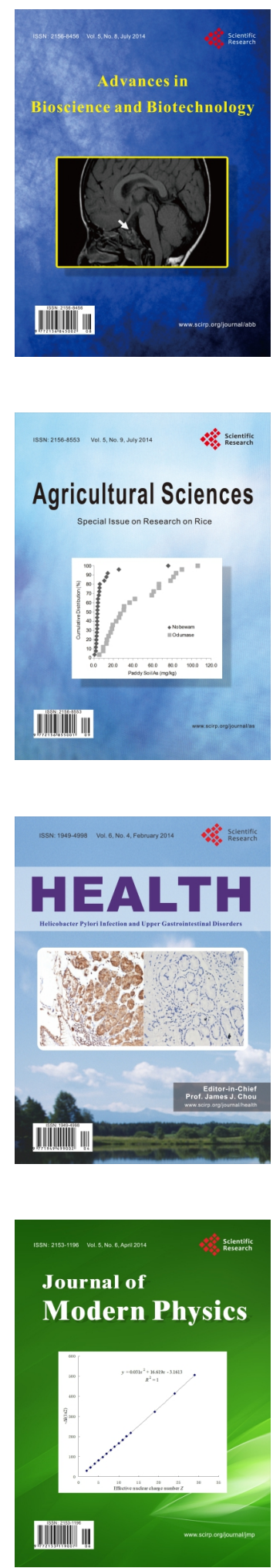
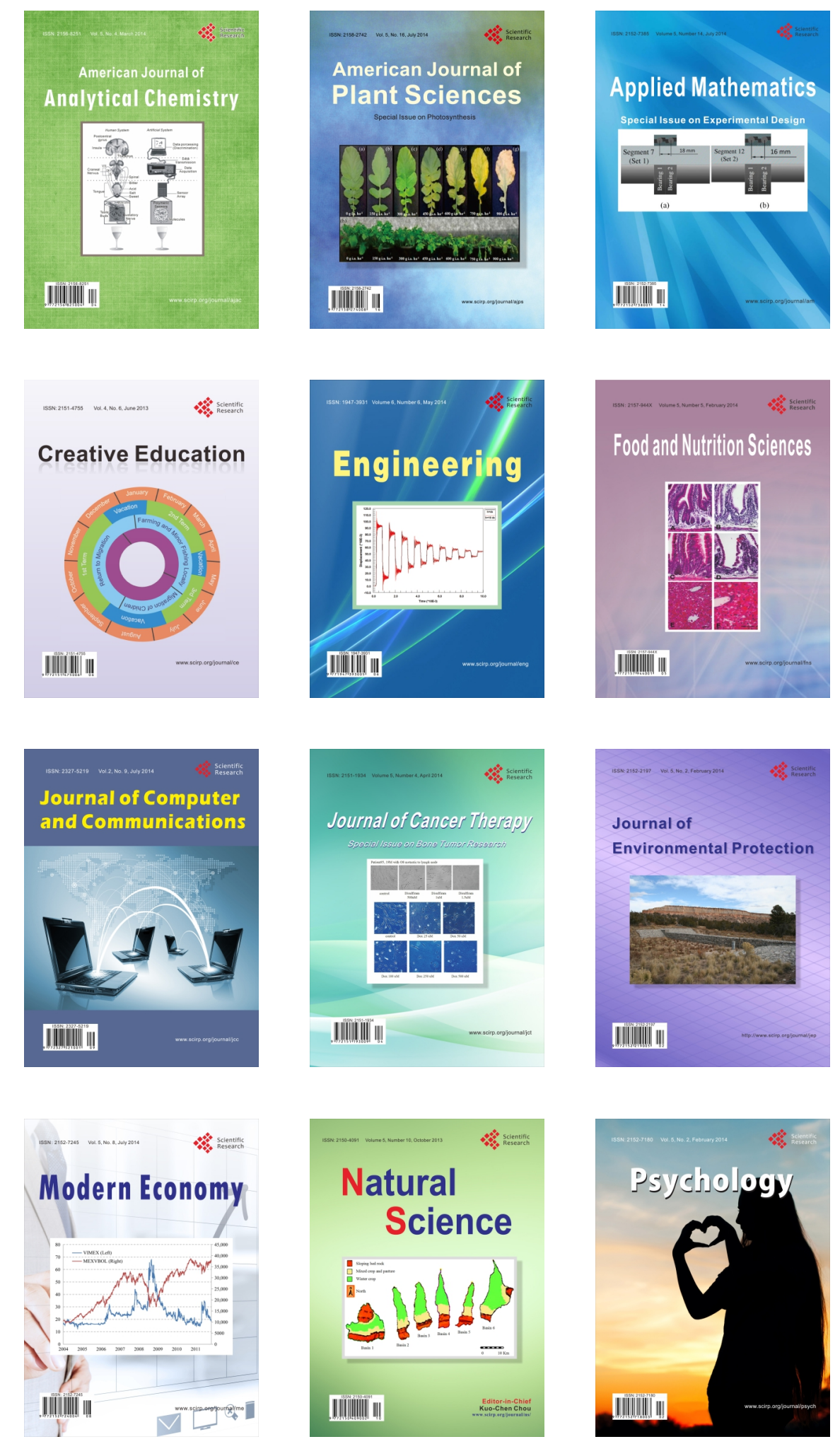